\title{
The genetic and regulatory architecture of ERBB3-type 1 diabetes susceptibility locus
}

\author{
Simranjeet Kaur ${ }^{\text {a, b }}$, Aashiq H. Mirza ${ }^{\text {a, b, c }}$, Caroline A. Brorsson a, Tina Fløyel ${ }^{\text {a }}$,

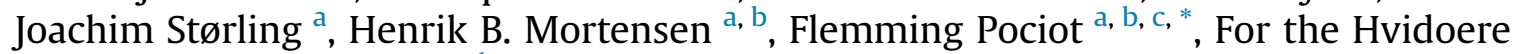 \\ International Study Group ${ }^{1}$ \\ a Copenhagen Diabetes Research Center (CPH-DIRECT), Department of Pediatrics, Herlev University Hospital, Herlev Ringvej 75, DK-2730 Herlev, Denmark \\ ${ }^{\mathrm{b}}$ Faculty of Health and Medical Sciences, University of Copenhagen, Denmark \\ c Center for Non-coding RNA in Technology and Health, University of Copenhagen, Denmark
}

\section{A R T I C L E I N F O}

\section{Article history:}

Received 22 July 2015

Received in revised form

29 September 2015

Accepted 1 October 2015

Available online 9 October 2015

\section{Keywords:}

ERBB3

CTCF

IncRNAs

Type 1 diabetes

Beta cell

Apoptosis

\begin{abstract}
A B S T R A C T
The study aimed to explore the role of ERBB3 in type 1 diabetes (T1D). We examined whether genetic variation of $E R B B 3$ (rs2292239) affects residual $\beta$-cell function in T1D cases. Furthermore, we examined the expression of ERBB3 in human islets, the effect of ERBB3 knockdown on apoptosis in insulinproducing INS-1E cells and the genetic and regulatory architecture of the ERBB3 locus to provide insights to how rs2292239 may confer disease susceptibility. rs2292239 strongly correlated with residual $\beta$-cell function and metabolic control in children with T1D. ERBB3 locus associated lncRNA (NONHSAG011351) was found to be expressed in human islets. ERBB3 was expressed and down-regulated by pro-inflammatory cytokines in human islets and INS-1E cells; knockdown of ERBB3 in INS-1E cells decreased basal and cytokine-induced apoptosis. Our data suggests an important functional role of $E R B B 3$ and its potential regulators in the $\beta$-cells and may constitute novel targets to prevent $\beta$-cell destruction in T1D.
\end{abstract}

() 2015 Elsevier Ireland Ltd. All rights reserved.

\section{Introduction}

Type 1 diabetes (T1D) is a multifactorial disease with both genetic and environmental determinants. Genome-wide association studies (GWAS) have identified around 50 genetic loci that contribute to disease susceptibility (Barrett et al., 2009; Onengut-Gumuscu et al., 2015) (www.t1dbase.org) but the underlying mechanisms largely remain unknown. Several studies identified association between T1D and variations at chromosome $12 \mathrm{q} 13$ surrounding the ERBB3 gene. Originally, association with T1D was demonstrated for the single nucleotide polymorphism (SNP) rs11171739, which maps between the RPS26 and ERBB3 genes (Burton et al., 2007). Most studies, including a meta-analysis, identified the strongest association with the single nucleotide polymorphism (SNP) rs2292239, located in intron 7 of ERBB3 (Barrett et al., 2009; Onengut-Gumuscu et al., 2015;

\footnotetext{
* Corresponding author. CPH-DIRECT, Department of Pediatrics, Herlev University Hospital, Herlev Ringvej 75, DK-2730 Herlev, Denmark.

E-mail address: flemming.pociot.01@regionh.dk (F. Pociot).

1 Members of the Hvidoere International Study Group are listed in the Appendix.

Burton et al., 2007; Hakonarson et al., 2008; Todd et al., 2007; Keene et al., 2012). Genetic variations in ERBB3 have also been used in different model approaches to improve prediction of T1D (Winkler et al., 2012; Bonifacio et al., 2014), autoimmunity (Törn et al., 2015; Brorsson and Pociot, 2015) and disease progression (Achenbach et al., 2013; Andersen et al., 2013). Despite the convincing genetic association of the ERBB3 region with T1D the functional implication underlying this has not been explored in detail, although it has been suggested that their most likely mechanism for affecting disease risk would be through effects on gene expression (Keene et al., 2012).

$E R B B 3$ is a member of the epidermal growth factor receptor (EGFR) family with impaired tyrosine kinase activity. It forms heterodimers with other EGFR family members that do have kinase activity thereby allowing activation of multiple signaling pathways including the phosphoinositol 3-kinase/AKT survival/mitogenic pathway. Increased ERBB3 expression plays an important role in the progression of several tumour forms (Baselga and Swain, 2009). Interestingly, the ERBB3 locus also overlaps with other autoimmune disease loci such as psoriasis, rheumatoid arthritis, vitiligo and alopecia. 


\section{Materials and methods}

\subsection{Patients}

This cross-sectional study involved 18 pediatric centres worldwide (De Beaufort et al., 2013). All children diagnosed with type 1 diabetes mellitus below 11 years of age and with diabetes duration of at least 1 year, treated at these centres, were invited to participate. Of 1209 eligible children in 18 centres, 1133 (93.7\%) children (47.4\% females; mean age: $8.0 \pm 2$ years; diabetes duration: $3.8 \pm 2.1$ years) participated and $1107(91.6 \%)$ provided a blood sample for HbA1c analysis and capillary blood spots for genetic analysis. Genotyping was possible on 873 samples (79\%). The children were characterized for gender, age at diagnosis, diabetes duration, HbA1c, insulin dose and insulin dose-adjusted HbA1c (IDAA1c) (calculated as HbA1c $+4^{*}$ insulin dose $/ \mathrm{kg} / 24 \mathrm{~h}$ ) as a surrogate marker for residual C-peptide and $\beta$-cell function (Max Andersen et al., 2014). For all children, detailed descriptions of the insulin regimens, episodes of severe hypoglycemia (seizures or loss of consciousness in the past 3 months), and diabetic ketoacidosis (DKA requiring hospital admission within the past 12 months) were obtained. A centralized HbA1c measurement was performed by the $\mathrm{TOSOH}^{\circledR}$ liquid chromatography (DCCT aligned, normal range: 4.4-6.3\%; IFFC: 25-45 $\mathrm{mmol} / \mathrm{mmol}$ ).

\subsection{DNA extraction and genotyping}

DNA was extracted from dried blood spots on filter paper using the DNeasy kit (Qiagen, Hilden, Germany) following the manufacturer's instruction. The SNP, rs2292239 in intron 7 of the ERBB3 gene, as well as the rs2187668 and rs7454108 of the MHC region, were genotyped using predesigned TaqMan ${ }^{\circledR}$ SNP Genotyping Assays (Life Technologies, Carlsbad, CA, USA). The two MHC SNPs were used to stratify for the HLA DR3/4 high-risk genotype (Barker et al., 2008; Romanos and Wijmenga, 2009). Linear regression models were used to test the association between metabolic control (HbA1c, insulin dose and IDAA1c) as dependent variables and SNP genotypes as the descriptive variables. Comparison between groups was performed using a two-tailed t test.

\subsection{Functional SNPS and cis-eQTLs}

To identify SNPs associated with changes in expression (ciseQTLS) of ERBB3, we integrated data from multiple eQTL studies (Stranger et al., 2012; Westra et al., 2013; GTEx Consortium, 2015). GWAS SNPs ( $p$-value $\leq 0.01$ ) for T1D were retrieved from T1DGC (Barrett et al., 2009) and linkage disequilibrium (LD) analysis was performed using SNAP (Johnson et al., 2008). We used ENCODE datasets (ChIP-Seq peaks, DNase I hypersensitivity peaks, DNase I footprints) from UCSC genome browser (Rosenbloom et al., 2013) (http://genome.ucsc.edu/) and RegulomeDB (Boyle et al., 2012) to identify functional evidence associated with ERBB3 variants.

\subsection{Culture and treatment of human islets}

Human islets were isolated from pancreata of four healthy organ donors provided through the integrated islet distribution program (IIDP) and cultured as described previously (Ardestani et al., 2014; Schulthess et al., 2009). Islets were exposed to $2 \mathrm{ng} / \mathrm{ml}$ recombinant human IL-1 $\beta$ (R\&D Systems, Minneapolis, MN, USA), $1000 \mathrm{U} / \mathrm{ml}$ recombinant human IFN- $\gamma$ (PeproTech, Rocky Hill, NJ, USA), and $1000 \mathrm{U} / \mathrm{ml}$ TNF $\alpha$ (R\&D Systems) for 6-72 h.

\subsection{Culture and transfection of INS-1E cells}

INS-1E cells were cultured as previously described (Fløyel et al., 2014). Cells were transfected with $30 \mathrm{nM}$ of siRNA. Lipofectamine RNAiMAX (Life Technologies) was used as transfectant. Nontargeting control siRNA (siCTRL) and siRNA against ERBB3 (siERBB3) were SmartPool siGENOME siRNAs (Dharmacon, Lafayette, CO, USA). Two days post transfection cells were treated with or without $150 \mathrm{pg} / \mathrm{mL}$ recombinant mouse IL-1 $\beta$ (BD Pharmingen, San Diego, CA, USA) and $5 \mathrm{ng} / \mathrm{mL}$ recombinant rat IFN $-\gamma$ (R\&D Systems) for $24 \mathrm{~h}$.

\subsection{Non-coding RNAs within ERBB3 locus}

NONCODE v4 database (Xie et al., 2014) was used to identify lncRNAs present within the ERBB3 locus. We used StarBase v2.0 (Li et al., 2014) to identify miRNA binding sites within ERBB3 $3^{\prime} \mathrm{UTR}$ based on CLIP-Seq overlap (using selection criteria for target prediction by at least two tools and $\geq 2$ CLIP-Seq experiments). Perturbation of miRNA binding within the $3^{\prime} \mathrm{UTR}$ of ERBB3 was predicted by miRdSNP (Bruno et al., 2012).

\subsection{Expression of ERBB3 and its associated lncRNAs in human islets and other tissues}

For expression of ERBB3 and its associated lncRNAs across 14 human tissues, we leveraged publicly available Human BodyMap (HBM) 2.0 RNAseq data (ENA archive: ERP000546) and NONCODE v4 (Xie et al., 2014). Expression values were expressed as fragments per kilobase of exon per million reads (FPKMs). The absolute value of the Pearson correlation coefficient was calculated for all pairwise comparisons of gene-expression values for ERBB3 and associated IncRNAs. All statistical analyses were performed with Bioconductor in the R statistical environment (Gentleman et al., 2004).

Total RNA was isolated from human islets using TriFast ${ }^{\mathrm{TM}}$ (Peqlab, Erlangen, Germany) following the manufacturer's instructions. cDNA was prepared using iScript ${ }^{\mathrm{TM}}$ cDNA synthesis kit (BioRad, Hercules, CA, USA), except for the antisense IncRNA NONHSAG011351, where a gene- and strand-specific reverse transcription was performed as described previously (Perocchi et al., 2007) using iScript ${ }^{\mathrm{TM}}$ Select cDNA synthesis kit (BioRad). Quantitative real-time PCR (qPCR) was performed on ERBB3, CTCF and three lncRNAs (NONHSAG011348, NONHSAG011349 and NONHSAG011351) using inventoried and custom-designed PrimeTime qPCR 5' Nuclease assays (Integrated DNA Technologies, Coralville, IA, USA) and Brilliant III Ultra-Fast QPCR Master Mix (Agilent Technologies, Santa Clara, CA, USA) with $10 \mathrm{ng} /$ well of cDNA as template. Primer sequences are provided in Supplementary Table S1. Expression was represented by deltaCT method with GAPDH as the reference gene. Additionally, expression of IncRNAs NONHSAG011348, NONHSAG011349, NONHSAG011351 and ERBB3 was also measured in HapMap lymphoblastoid cell lines of individuals of European descent (CEU, $\mathrm{n}=50$ ) (Fløyel et al., 2014).

\subsection{Western blotting}

Human islets were washed with PBS and lysed in RIPA lysis buffer containing $50 \mathrm{mM}$ Tris-HCL pH 8.0, $150 \mathrm{mM} \mathrm{NaCl}, 1 \% \mathrm{NP}-40$, $0.5 \%$ sodium deoxycholate, $0.1 \%$ SDS supplemented with $1 \mathrm{X}$ Protease- and Phosphatase-inhibitors (100X) (Pierce, Rockford, IL, USA). INS-1E cells were lysed in M-PER Mammalian Protein Extraction Reagent supplemented with Halt Protease \& Phosphatase Inhibitor Cocktail (all from Pierce). Western blotting was performed as previously described (Fløyel et al., 2014). Antibodies used were anti-ERBB3 (12708, Cell Signaling, Danvers, MA, USA), anti- 
cleaved caspase-3 (9661, Cell Signaling), anti- $\alpha$-tubulin (T8203, Sigma-Aldrich, St. Louis, MO, USA), anti-GAPDH (ABS16, Millipore, Billerica, MA, USA), anti-rabbit IgG (7074, Cell Signaling), and antimouse IgG (7076, Cell Signaling).

\subsection{Apoptosis}

Apoptosis was determined using Cell Death Detection ELISA (Roche, Basel, Switzerland) as previously described (Fløyel et al., 2014). The activities of caspases 3 and 7 were measured by the Caspase-GLO 3/7 assay (Promega, Madison, WI, USA) using $50 \mu \mathrm{l}$ Caspase-GLO reagent per well. Both assays were measured on a M200-pro multiplate reader (Tecan, Männedorf, Switzerland). Data was calculated as fold over non-treated siCTRL-transfected cells.

\section{Results}

\section{1. rs2292239 associates with residual $\beta$-cell function and metabolic control}

The T1D-associated SNP rs2292239 was significantly associated with IDAA1c ( $p$-value $=0.0067)$ and HbA1c ( -value $=0.019)$ in a recessive model corrected for gender, age at diagnosis and diabetes duration (Table 1). The TT genotype carriers had a lower IDAA1c and a lower HbA1c compared to carriers of the GG and GT genotypes. No association to hypoglycemic events or DKA was observed. HLA DR3/ DR4 heterozygosity was associated with lower age at diagnosis (est. $=-0.45$; p-value $=0.007$ ). The DR3/DR4 genotype did not affect any measures of metabolic control. No significant differences in gender, age at diagnosis, disease duration, HbA1c, IDAA1c or insulin dose were observed between the genotyped (984/1133) and non-genotyped parts of the cohort.

\section{2. rs2292239, rs3741499 and rs4759229 are putatively functional}

To investigate how rs2292239 may confer disease susceptibility we explored the genetic and regulatory architecture of the ERBB3 locus. SNPs that act as cis-eQTLs, overlap ENCODE regulatory features and are in strong LD with a known disease-associated SNP are considered as functional SNPs (Schaub et al., 2012). rs2292239 has been shown to act as cis-eQTL for ERBB3 in whole blood (Westra et al., 2013; GTEx Consortium, 2015) and also for the neighboring genes SUOX (thyroid), RAB5B (skeletal muscle) and AC034102.1 (skeletal muscle) (Supplementary Fig. 1). We explored the ENCODE datasets for potential functional evidence of rs2292239 and found ChIP-Seq based evidence of a CTCF (CCCTC-binding factor) transcription factor (TF) binding site overlapping rs2292239 in multiple ENCODE cell lines (Fig. 1). In total, we found five CTCF binding sites within ERBB3, of which three had a conserved motif with known position weight matrices (PWMs) and DNase I footprint overlap (Fig. 1). These conserved CTCF motif binding sites were located within the promoter, $5^{\prime}$ UTR and the second exon of ERBB3 (Fig. 1).

Interestingly, in the HapMap3 CEU population rs2292239 was found to be in strong $\operatorname{LD}\left(\mathrm{r}^{2}=0.98\right)$ with rs3741499 and rs4759229 (Supplementary Fig. S2) and multiple lines of evidence based on ENCODE regulatory features suggested functionality for these two SNPs. For both SNPs, we observed enrichment for H3K4Me1 and H3K27Ac histone marks, which are often found near active regulatory elements (Supplementary Fig. S3). Additionally, rs4759229 overlapped a DNase I hypersensitivity peak and both rs4759229 and rs3741499 overlapped ChIP-Seq peaks for several TFs in multiple ENCODE cell lines (Supplementary Fig. S3). Intriguingly, we also found that rs4759229 lies within a known enhancer element (chr12:56474601-56474862) based on FANTOM5 human active enhancer atlas (Andersson et al., 2014). Taken together, these data indicate that rs2292239, rs3741499, and rs4759229 might be functional SNPs with regulatory implications.

\subsection{ERBB3 knockdown decreases basal and cytokine-induced apoptosis}

ERBB3 has been reported to be expressed and down-regulated in response to pro-inflammatory cytokines in human islets at the transcriptional level (Eizirik et al., 2012). Using western blotting we confirmed that ERBB3 is expressed and down-regulated by a mixture of IL- $1 \beta$, IFN $-\gamma$ and TNF $\alpha$ in human islets (p-value $<0.05$, Fig. $2 \mathrm{~A}$ ) and to IL-1 $\beta+$ IFN- $\gamma$ in INS-1E cells ( $p$-value $<0.05$, Fig. $2 \mathrm{~B}$ ).

To investigate whether ERBB3 is involved in $\beta$-cell apoptosis, we transfected INS-1E cells with non-targeting control siRNA or siRNA against ERBB3 and examined the effect on cytokine-induced apoptosis. To confirm siRNA-mediated knockdown of ERBB3, the protein expression of ERBB3 was examined. The ERBB3 expression was reduced by $50 \%$ compared to cells transfected with control siRNA (p-value $<0.05$, Fig. 3A). Importantly, knockdown of ERBB3 decreased basal and cytokine-induced apoptosis compared to cells transfected with control siRNA ( $\mathrm{p}$-value $<0.05$, Fig. 3B). These findings were supported by data showing decreased basal and cytokine-induced caspase 3 and 7 activities, as assessed by caspase activity assay ( $p$-value $<0.05$, Fig. $3 \mathrm{C}$ ) and western blotting ( $\mathrm{p}$ value $<0.05$, Fig. $3 D$ ), suggesting that $E R B B 3$ is required for apoptosis in INS-1E cells.

\subsection{Non-coding RNA regulation of the ERBB3 locus}

Non-coding RNAs (ncRNAs), e.g. IncRNAs and microRNAs (miRNAs) are known to play an important role in regulation of gene expression and their dysregulation has been associated with various human diseases, including T1D (Guay and Regazzi, 2013;

Table 1

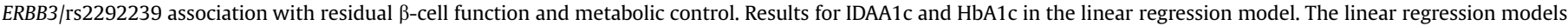
were corrected for gender, age at diagnosis and diabetes duration which were significantly associated with IDAA1c and insulin dose.

\begin{tabular}{|c|c|c|c|c|c|c|}
\hline \multirow{2}{*}{ Variable } & \multicolumn{2}{|l|}{ IDAA1c } & \multicolumn{2}{|l|}{ HbA1c (\%) } & \multicolumn{2}{|c|}{ Insulin dose $(\mathrm{U} / \mathrm{kg})$} \\
\hline & Estimate (S.E.) & P-value & Estimate (S.E.) & P-value & Estimate (S.E.) & P-value \\
\hline Gender (male) & $-0.26(0.10)$ & 0.014 & $-0.09(0.07)$ & 0.19 & $-0.0436(0.02)$ & 0.0077 \\
\hline Age at diagnosis & $0.05(0.03)$ & 0.051 & $-0.001(0.02)$ & 0.95 & $0.0131(0.004)$ & 0.0021 \\
\hline Diabetes duration & $0.19(0.03)$ & $1.31 \times 10^{-09}$ & $0.04(0.02)$ & 0.052 & $0.0360(0.004)$ & $1.12 \times 10^{-13}$ \\
\hline \multicolumn{7}{|l|}{ rs2292239 genotype model } \\
\hline GG (N = 343) (39\%; 1000 Genomes: 46\%) & Reference & - & Reference & - & Reference & - \\
\hline GT (N = 406) (47\%; 1000 Genomes: 41\%) & $-0.05(0.11)$ & 0.67 & $-0.03(0.08)$ & 0.66 & $-0.0037(0.02)$ & 0.83 \\
\hline $\begin{array}{l}\text { TT }(N=124)(14 \% ; 1000 \text { Genomes: } 13 \%) \\
\text { rs2292239 recessive model }\end{array}$ & $-0.43(0.16)$ & 0.0077 & $-0.26(0.11)$ & 0.020 & $-0.042(0.03)$ & 0.093 \\
\hline (TT vs. GG + GT) & $-0.40(0.15)$ & 0.0067 & $-0.24(0.10)$ & 0.019 & $-0.040(0.02)$ & 0.083 \\
\hline
\end{tabular}




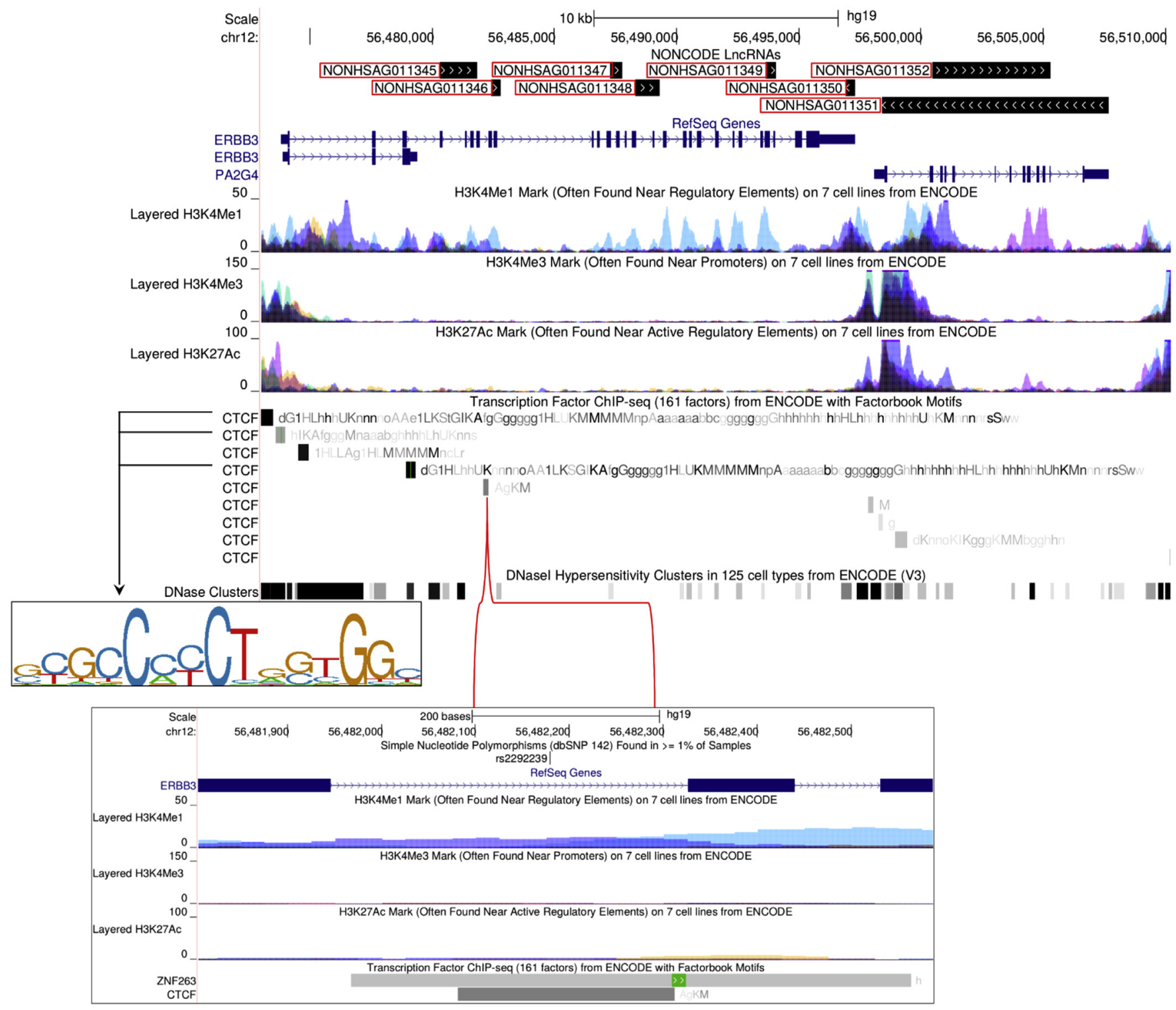

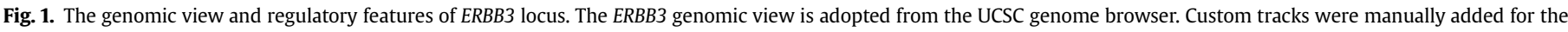

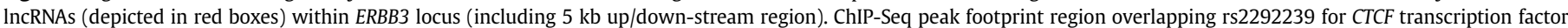

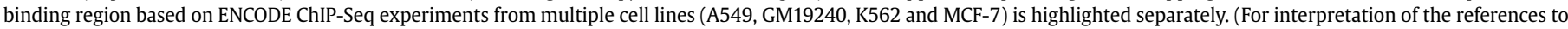
color in this figure legend, the reader is referred to the web version of this article.)

Hezova et al., 2010; Pullen and Rutter, 2012). Sequence variations within IncRNAs and miRNAs have been linked with their expression dysregulation (Guay and Regazzi, 2013; Mirza et al., 2014). We identified five sense IncRNAs (NONHSAG011345, NONHSAG011346, NONHSAG011347, NONHSAG011348 and NONHSAG011349) and one antisense lncRNA (NONHSAG011350) intersecting the ERBB3 gene (Fig. 1). However, NONHSAG011350 was excluded from further analysis due to lack of evidence in Gencode annotations (Harrow et al., 2012). Additionally, in $5 \mathrm{~kb}$ up/down-stream vicinity of ERBB3, we found two lncRNAs (antisense NONHSAG011351 and sense NONHSAG011352) intersecting the neighboring PA2G4 gene (Fig. 1). All lncRNAs were found to be highly conserved based on sequence alignments with three primates (chimpanzee, orangutan, and macaque) (data not shown).

As SNPs within or up-/down-stream of a miRNA binding site can alter the efficiency of miRNA binding and result in gene dysregulation (Bruno et al., 2012), we explored CLIP-Seq datasets from
starBase database v2.0 to identify miRNA binding sites within ERBB3. We found eight miRNAs that target the ERBB3 $3^{\prime} \mathrm{UTR}$ of which four were altered by up-/down-stream SNPs and indels (Supplementary Table S2). However, none of our candidate SNPs affected the miRNA binding.

\subsection{Expression of ERBB3 and its associated lncRNAs}

On comparing the expression profiles of the lncRNAs with ERBB3 across an array of 14 tissues using Human BodyMap (HBM) 2.0 RNAseq data, we observed distinct tissue-specific expression patterns (Fig. 4A). The intronic/exonic IncRNAs (NONHSAG011345, NONHSAG011346, NONHSAG011347, NONHSAG011348 and NONHSAG011349) exhibited relatively lower expression as compared to the ERBB3. The lncRNAs intersecting PA2G4 (NONHSAG011351 and NONHSAG011352) were found to be expressed across most of the HBM tissues at similar levels as ERBB3. The expression profiles of 

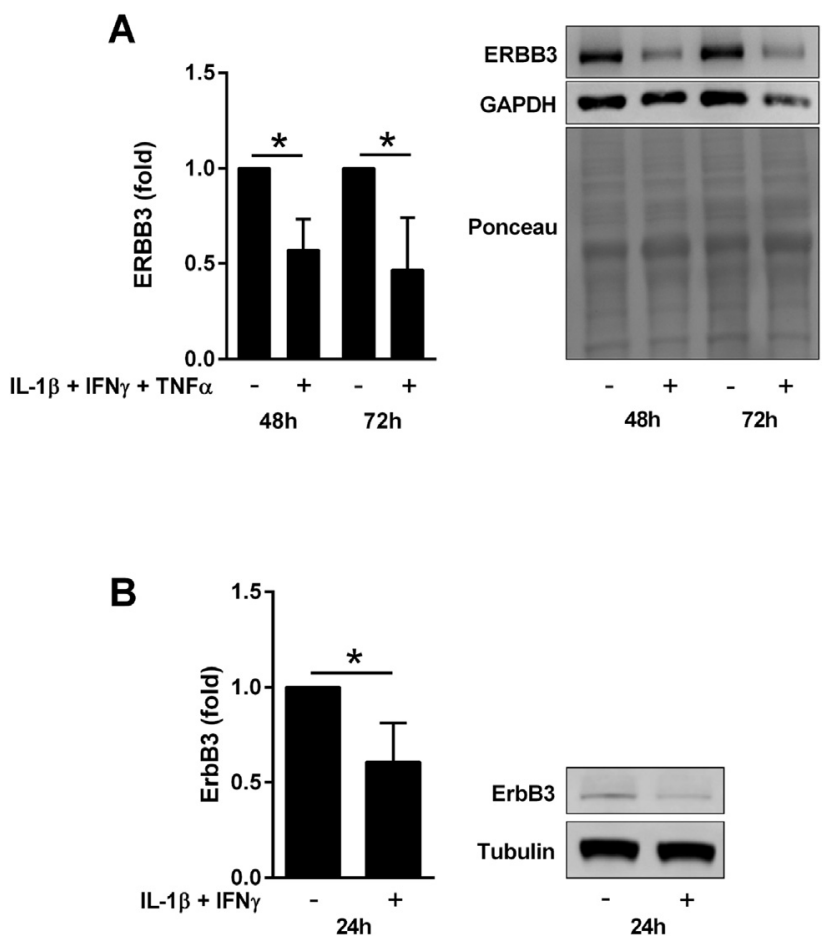

Fig. 2. Cytokine-mediated down-regulation of ERBB3 in human islets and INS-1E cells A) ERBB3 protein expression in human islets $(n=3)$ left untreated or exposed to IL-1 $\beta$ IFN- $\gamma$ and TNF $\alpha$ for 48 or 72 h. B) ERBB3 protein expression in INS-1E cells $(n=4)$ left untreated or exposed to IL-1 $\beta$ and IFN- $\gamma$ for $24 \mathrm{~h}$. Data are mean \pm SD. ERBB3 expression was normalized to ponceau stainings (A) or $\alpha$-Tubulin (B). * ${ }^{*}$ p $<0.05$.

ERBB3 and its associated lncRNAs evidently showed high tissuespecificity. We next calculated the absolute pairwise correlations (Pearson) between the expression levels of ERRB3 and its associated IncRNAs (Supplementary Fig. S4). The highest correlations were observed for intronic/exonic lncRNAs ranging from 0.57 to 0.91 . The correlations between the different lncRNAs ranged from 0.1 to 0.96 . Strong correlations were also observed between PA2G4 and its intersecting lncRNAs NONHSAG011351 $(\mathrm{r}=0.8)$ and NONHSAG011352 ( $\mathrm{r}=0.5)$ (Supplementary Fig. S5).

We examined the expression of ERBB3, CTCF, two intronic/exonic IncRNAs (NONHSAG011348 and NONHSAG011349) and the antisense IncRNA(NONHSAG011351) in four human islet preparations. As shown in Fig. $4 \mathrm{~B}$, ERBB3 and CTCF were expressed at similar levels, whereas the antisense IncRNA NONHSAG011351 was expressed at a higher level. The intronic/exonic IncRNAs NONHSAG011348 and NONHSAG011349 were not found to be expressed in the human islets.

Additionally, we measured the expression of lncRNAs NONHSAG011348, NONHSAG011349, NONHSAG011351 and ERBB3 in HapMap cell lines (CEU population) carrying both heterozygous and homozygous genotypes for SNPs rs2292239, rs3741499, and rs4759229. The genotypic effect of these SNPs was computed based on linear regression. Additionally, rs705708 was included in this analysis based on results from our recent study which provided regulatory evidence for rs705708 and its potential structuredisruptive effects on NONHSAG011348 (Mirza et al., 2014). We found that rs705708 had a cis-eQTL effect on both ERBB3 (pvalue $<0.05$ ) and antisense IncRNA NONHSAG011351 (pvalue $<0.05$ ). The GG genotype associated with lower expression of ERBB3 and NONHSAG011351 (Fig. 5).

\subsection{ERBB3 interacts with other T1D candidate genes}

The EGFR signaling pathway has been well studied with respect to protein-protein interactions. Although the interaction partners for members of the ERBB family have been identified, their functional roles are not well elucidated (Olayioye et al., 2000). We retrieved all known protein-protein interacting partners for ERBB3 using BioGRID (Stark et al., 2006); a database of protein and genetic interactions based on high- and low-throughput experiments. Overall, we found 88 physical associations for 61 interacting partners for ERBB3 (Supplementary Fig. S6). Intriguingly, members of the ERBB3 protein interactome included 6 bona-fide T1D candidate genes: ERBB2, GRB7, PA2G4, PTPN11, TENC1 and SH2B3, all known to be regulated by pro-inflammatory cytokines (Eizirik et al., 2012). All six genes were significantly enriched within the ERBB3 interactome (p-value $=7.93 \mathrm{e}-04$, binomial test). Noteworthy, two direct ERBB3 interactors PA2G4 and RNF41 share the same locus as ERBB3 (12q13.2), which suggests a regulatory interplay both at genetic and protein levels.

\section{Discussion}

We show that genetic variation at the ERBB3 locus (rs2292239) is associated with residual $\beta$-cell function, as assessed by IDAA1c, and metabolic control in young children with T1D and disease duration of at least 1 year. The TT genotype carriers had a better residual $\beta$-cell function and a better metabolic control compared to carriers of the GG + GT genotype. A recent study by Torn $C$ et al. (Törn et al., 2015) showed that the rs2292239 is also associated with T1D specific autoantibody production. Autoantibody measurements were not available in the present study, but we have recently shown that rs2292239 is associated with multiple autoantibodies in longstanding T1D (Brorsson and Pociot, 2015). We could not replicate the finding that rs2292239 was associated with a lower age at onset (Hakonarson et al., 2008; Espino-Paisan et al., 2011), most likely due to the fact that all children in our cohort were diagnosed before the age of 11 years.

Although ERBB3 is a known T1D candidate gene, the exact role of $E R B B 3$ in the pathogenesis of T1D remains largely obscure. Our data show that ERBB3 is a novel regulator of $\beta$-cell apoptosis, i.e. ERBB3 is down regulated by cytokines and knockdown of ERBB3 reduces basal and cytokine-induced apoptosis. The PI3K/Akt pathway constitutes an important general survival pathway including in $\beta$-cells (Elghazi and Bernal-Mizrachi, 2009). As ERBB3 has been shown to be associated with increased PI3K/Akt signaling (Lee et al., 2009, 2014a) and inhibition of Bak/Bax-mediated mitochondrial apoptosis (Lee et al., 2014b), we hypothesize that the observed protective effect on caspase 3/7 activation in INS-1E cells is due to altered signaling via PI3K/Akt and/or mitochondrial apoptosis signaling.

Previous studies suggest that ERBB3 contributes to disease susceptibility by modulating antigen presenting cell function (Wang et al., 2010) and autoimmunity (Törn et al., 2015; Brorsson and Pociot, 2015) indicating dual functional roles for ERBB3 during the pathogenesis of T1D. The role of ERBB3 in T1D should be examined further in cellular and animal models to gain insights into the underlying molecular mechanisms. The ERBB3 locus exhibits a relatively high degree of sequence variability (34 variations/ $1 \mathrm{~Kb}$ ) including SNPs, indels and other somatic mutations. Some of these variations could be of functional significance particularly those that lie within the binding sites of known TFs. Generally, functional TF binding is enriched at sites that are enriched in active enhancers, regulatory elements harboring multiple TF binding sites, and at sites with predicted higher binding affinity (Andersson et al., 2014; Cusanovich et al., 2014). Our analysis points towards the functional significance of SNPs rs2292239, rs3741499 and rs4759229 based on the overlap with an active enhancer element, CLIP-Seq peaks and DNase I footprints coverage, and the existence 
A
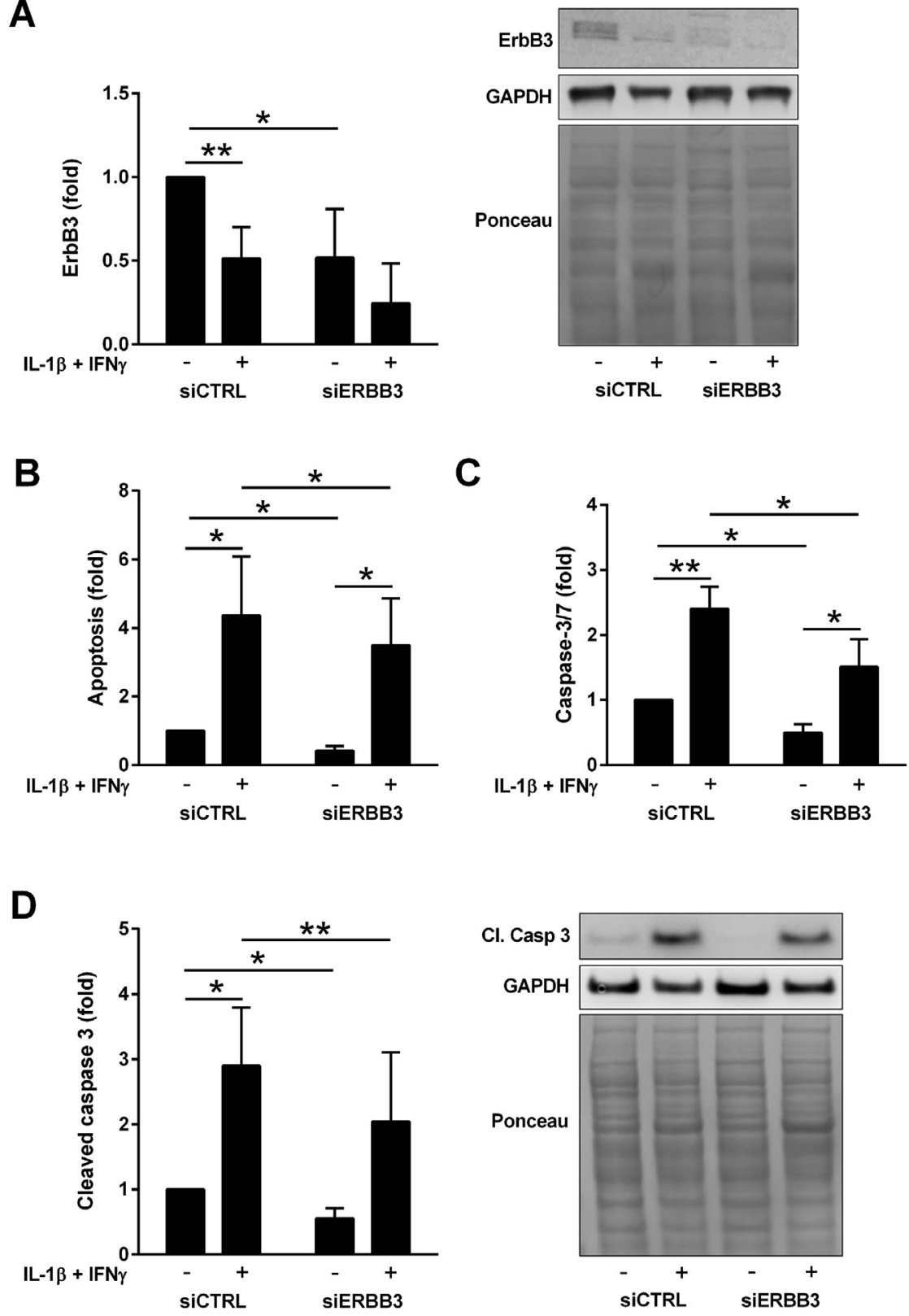

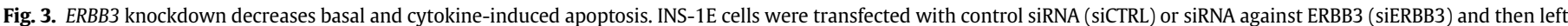

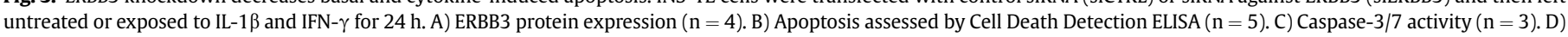
Cleaved caspase-3 protein expression $(n=4)$. Data are mean and SD. Protein expression was not normalized. ${ }^{*}: p<0.05 .{ }^{* *}: \mathrm{p}<0.01$.

of multiple TF binding sites. Interestingly, rs2292239 also affects the expression of ERBB3 and vicinity genes (SUOX and $R A B 5 B$ ) in various tissues, suggesting an even more complex impact on disease susceptibility of this locus.

Evolutionary sequence conservation and distinct tissue-specific expression patterns of ERBB3 locus associated lncRNAs indicates their potential regulatory significance. LncRNAs are in general expressed in a tissue-specific manner and comparatively at lower levels than their protein-coding counterparts (Derrien et al., 2012). Furthermore, IncRNAs are known to regulate expression of both proximal (cis) and distal (trans) protein-coding genes (Kornienko et al., 2013). In particular, antisense IncRNAs are involved in transcription regulation of protein-coding genes in cis (Wilusz et al., 2009; Xu et al., 2011). Our results suggest potential regulatory roles of the antisense lncRNA NONHSAG011351 on ERBB3 in human islets.
CTCF is a ubiquitously expressed transcription factor that is known to bind across the genome to chromatin boundaries, enhancers and gene promoters to regulate their transcriptional activities. CTCF has been found to be up-regulated by high glucose and insulin dosage in pancreatic islet $\beta$-cells where it mediates effects of glucose on $\beta$-cell survival (Tsui et al., 2014). The presence of CTCF binding sites in the $5^{\prime}$ UTR and introns of genes suggests a role for $C T C F$ in regulating transcriptional events downstream of the initiation step (Chen et al., 2012). ChIP-Seq signals, together with the DNase I footprints evidence indicates that CTCF might have a pivotal regulatory role in controlling ERBB3 expression by binding to its promoter and intragenic (exon/intron) region through a canonical conserved CTCF binding site motif. Nevertheless, our results warrant a systematic experimental follow-up to explore and validate the potential regulatory roles of CTCF and the IncRNAs in 


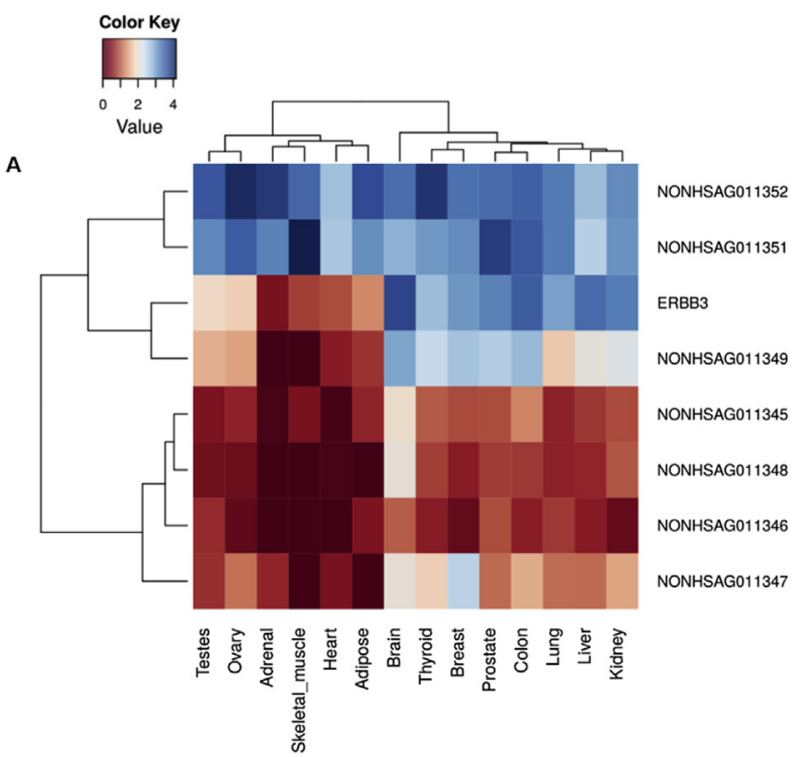

B

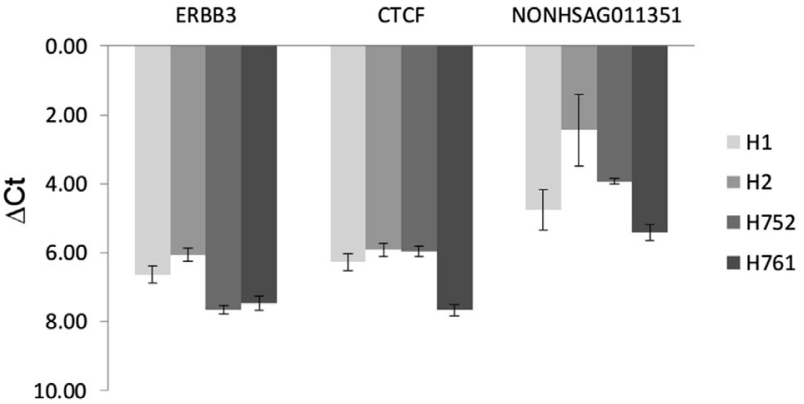

. 4. Expression of $E R B B 3$ and its associated IncRNAs in human islets and other tissues. (A) Heatmap and hierarchical clustering of expression levels (log 2 FPKM) for $E R B B 3$ and its associated lncRNAs across 14 tissues. (B) qPCR results for ERBB3, CTCF and antisense IncRNA NONHSAG011351 in human islets from four donors. The legend on the right represents the four islet preparations.

HapMap (CEU) eQTL rs705708 ERBB3

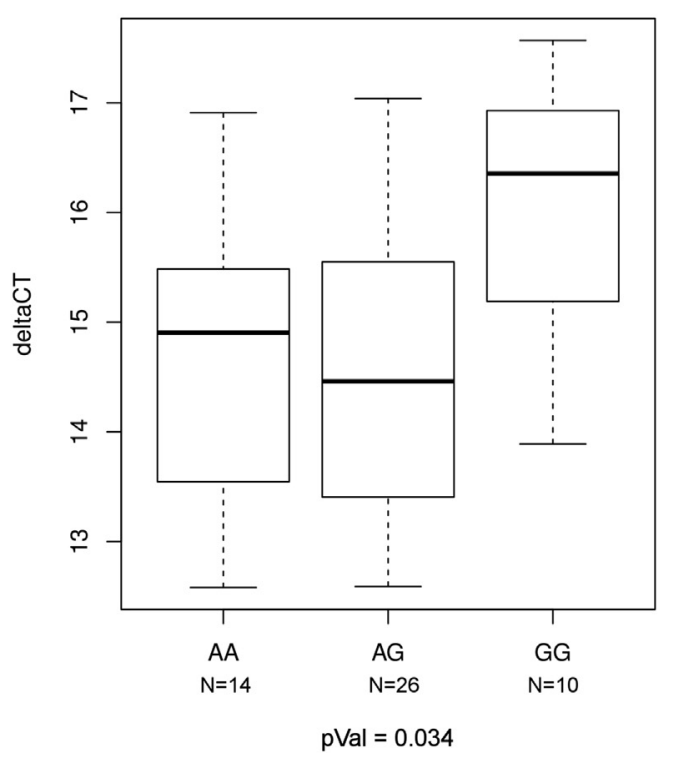

HapMap (CEU) eQTL rs705708 NONHSAG011351

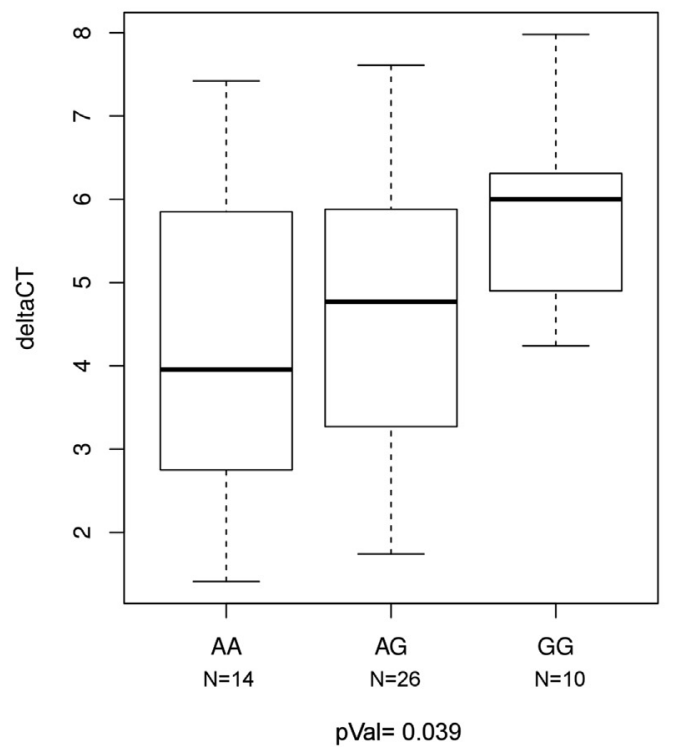

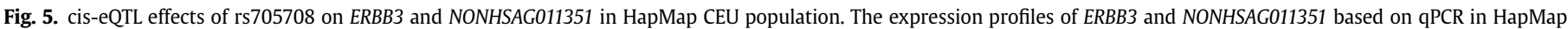
(CEU) population. The cis-eQTLs were calculated for rs705708 based on linear regression.

modulating ERBB3 expression.

\section{Acknowledgments}

SK was supported by a fellowship from University of Copenhagen. AHM was supported by a fellowship from the Danish Council for Strategic Research "Center for non-coding RNA in Technology and Health" (09-067036/DSF). TF and FP were supported by the European Foundation for the Study of Diabetes. In addition, this work was supported by grants to FP from the National Institute of Health (1 DP3 DK085678) and to SK and AHM from the Poul and Erna Sehested Hansen Foundation. We would like to thank Novo
Nordisk for supporting The Hvidoere Study Group on Childhood Diabetes and Fie Hillesø for expert technical assistance. We also would like to thank Federico Paroni and Kathrin Maedler for providing RNA from human islets.

\section{Appendix}

Members of the Hvidoere Study Group on Childhood Diabetes who have contributed to the Hvidoere Study in Young Children:

Henk-Jan Aanstoot, Center for Pediatric and Adolescent Diabetes Care and Research, Rotterdam, The Netherlands; Carine E de Beaufort, Pediatric Clinic, Centre Hospitalier de Luxembourg, 
Luxembourg, GD de Luxembourg; Fergus Cameron, Department of Endocrinology and Diabetes, Royal Children's Hospital, Parkville, Victoria, Australia; Luis Castano, Endocrinology and Diabetes Research Group, Hospital de Cruces, University of Basque Country, Barakaldo, Spain; Harry Dorchy, Diabetology Clinic, University Hospital Reine Fabiola, Brussels, Belgium; Lynda K Fisher, Department of Endocrinology and Diabetes Children's Hospital of Los Angeles, Los Angeles, CA, USA; Eero Kaprio, Department of Paediatrics, Peijas Hospital, HUS, Finland; Karin Lange, Department of Medical Psychology, Hannover Medical School, Hannover, Germany; Andreas Neu, Clinic for Children and Adolescence, University of Tuebingen, Tuebingen, Germany; Pal R Njolstad, Department of Clinical Medicine University of Bergen, Bergen, Norway \& Department of Paediatrics, Haukeland University Hospital, Bergen, Norway; Moshe Phillip, National Center of Childhood Diabetes, Schneiders Medical Center of Israel, Petah Tikva, Israel; Jean J Robert, Department of Childhood and Adolescent Diabetes, Hôpital Necker-Enfants Malades, Paris, France; Tatsuhiko Urukami, School of Medicine, Nihon University, Tokyo, Japan; Tim Barrett, Institute of Child Health and Birmingham Children's Hospital, University of Birmingham, Birmingham, UK; Francesco Chiarelli, Clinica Pediatrica, Ospedale Policlinico, Chieti, Italy; Thomas Danne, Kinderkrankenhaus auf der Bult, Hannover, Germany; Hilary Hoey, University of Dublin, National Children's Hospital, Tallaght, Ireland; Mirjana Kocova, Pediatric Clinic-Skopje, Republic of Macedonia; Henrik B. Mortensen, Department of Pediatrics, Herlev Hospital \& Faculty of Health and Medical Sciences, University of Copenhagen, Denmark; Eugen J. Schoenle, University Children's Hospital, Zurich, Switzerland; Peter GF Swift, Leicester Royal Infirmary Childrens Hospital, Leicester, UK; Maurizio Vanelli, Clinica Pediatrica, Centro di Diabetologia, Parma, Italy; Jan Åman, Örebro Medical Centre Hospital, Department of Paediatrics, Sweden.

\section{Appendix A. Supplementary data}

Supplementary data related to this article can be found at http:// dx.doi.org/10.1016/j.mce.2015.10.002.

\section{References}

Achenbach, P., Hummel, M., Thümer, L., et al., 2013. Characteristics of rapid vs slow progression to type 1 diabetes in multiple islet autoantibody-positive children. Diabetologia 56, 1615-1622.

Andersen, M.L.M., Rasmussen, M.A., Pörksen, S., et al., 2013. Complex multi-block analysis identifies new immunologic and genetic disease progression patterns associated with the residual $\beta$-cell function 1 year after diagnosis of type 1 diabetes. PLoS One 8, e64632.

Andersson, R., Gebhard, C., Miguel-Escalada, I., et al., 2014. An atlas of active enhancers across human cell types and tissues. Nature 507, 455-461.

Ardestani, A., Paroni, F., Azizi, Z., et al., 2014. MST1 is a key regulator of beta cell apoptosis and dysfunction in diabetes. Nat. Med. 20, 385-397.

Barker, J.M., Triolo, T.M., Aly, T.A., et al., 2008. Two single nucleotide polymorphisms identify the highest-risk diabetes HLA genotype: potential for rapid screening. Diabetes 57, 3152-3155.

Barrett, J.C., Clayton, D.G., Concannon, P., et al., 2009. Genome-wide association study and meta-analysis find that over 40 loci affect risk of type 1 diabetes. Nat. Genet. 41, 703-707.

Baselga, J., Swain, S.M., 2009. Novel anticancer targets: revisiting ERBB2 and discovering ERBB3. Nat. Rev. Cancer 9, 463-475.

Bonifacio, E., Krumsiek, J., Winkler, C., et al., 2014. A strategy to find gene combinations that identify children who progress rapidly to type 1 diabetes after islet autoantibody seroconversion. Acta Diabetol. 51, 403-411.

Boyle, A.P., Hong, E.L., Hariharan, M., et al., 2012. Annotation of functional variation in personal genomes using RegulomeDB. Genome Res. 22, 1790-1797.

Brorsson, C.A., Pociot, F., Type 1 Diabetes Genetics Consortium, 2015. Shared genetic basis for type 1 diabetes, islet autoantibodies, and autoantibodies associated with other immune-mediated diseases in families with type 1 diabetes. Diabetes Care 38 (Suppl. 3), 1-6. http://dx.doi.org/10.2337/dcS15-2003.

Bruno, A.E., Li, L., Kalabus, J.L., et al., 2012. miRdSNP: a database of diseaseassociated SNPs and microRNA target sites on $3^{\prime}$ UTRs of human genes. BMC Genomics 13, 44.
Burton, P.R., Clayton, D.G., Cardon, L.R., et al., 2007. Genome-wide association study of 14,000 cases of seven common diseases and 3000 shared controls. Nature 447, 661-678.

Chen, H., Tian, Y., Shu, W., et al., 2012. Comprehensive identification and annotation of cell type-specific and ubiquitous CTCF-binding sites in the human genome. PLoS One 7, e41374.

Cusanovich, D.A., Pavlovic, B., Pritchard, J.K., Gilad, Y., 2014. The functional consequences of variation in transcription factor binding. PLoS Genet. 10, e1004226.

De Beaufort, C.E., Lange, K., Swift, P.G.F., et al., 2013. Metabolic outcomes in young children with type 1 diabetes differ between treatment centers: the Hvidoere study in young children 2009. Pediatr. Diabetes 14, 422-428.

Derrien, T., Johnson, R., Bussotti, G., et al., 2012. The GENCODE v7 catalog of human long noncoding RNAs: analysis of their gene structure, evolution, and expression. Genome Res. 22, 1775-1789.

Eizirik, D.L., Sammeth, M., Bouckenooghe, T., et al., 2012. The human pancreatic islet transcriptome: expression of candidate genes for type 1 diabetes and the impact of pro-inflammatory cytokines. PLoS Genet. 8, e1002552.

Elghazi, L., Bernal-Mizrachi, E., 2009. Akt and PTEN: beta-cell mass and pancreas plasticity. Trends Endocrinol. Metab. 20, 243-251.

Espino-Paisan, L., de la Calle, H., Fernández-Arquero, M., et al., 2011. Polymorphisms in chromosome region 12q13 and their influence on age at onset of type 1 diabetes. Diabetologia 54, 2033-2037.

Fløyel, T., Brorsson, C., Nielsen, L.B., et al., 2014. CTSH regulates $\beta$-cell function and disease progression in newly diagnosed type 1 diabetes patients. Proc. Natl. Acad. Sci. U. S. A. 111, 10305-10310.

Gentleman, R.C., Carey, V.J., Bates, D.M., et al., 2004. Bioconductor: open software development for computational biology and bioinformatics. Genome Biol. 5, R80. http://dx.doi.org/10.1186/gb-2004-5-10-r80.

GTEx Consortium, 2015. Human genomics. The genotype-tissue expression (GTEx) pilot analysis: multitissue gene regulation in humans. Science 348 (6235), 648-660.

Guay, C., Regazzi, R., 2013. Circulating microRNAs as novel biomarkers for diabetes mellitus. Nat. Rev. Endocrinol. 9, 513-521.

Hakonarson, H., Qu, H.-Q., Bradfield, J.P., et al., 2008. A novel susceptibility locus for type 1 diabetes on Chr12q13 identified by a genome-wide association study. Diabetes 57, 1143-1146.

Harrow, J., Frankish, A., Gonzalez, J.M., et al., 2012. GENCODE: the reference human genome annotation for the ENCODE Project. Genome Res. 22, 1760-1774.

Hezova, R., Slaby, O., Faltejskova, P., et al., 2010. microRNA-342, microRNA-191 and microRNA-510 are differentially expressed in T regulatory cells of type 1 diabetic patients. Cell. Immunol. 260, 70-74.

Johnson, A.D., Handsaker, R.E., Pulit, S.L., et al., 2008. SNAP: a web-based tool for identification and annotation of proxy SNPs using HapMap. Bioinformatics 24, 2938-2939.

Keene, K.L., Quinlan, A.R., Hou, X., et al., 2012. Evidence for two independent associations with type 1 diabetes at the 12 q13 locus. Genes Immun. 13, 66-70.

Kornienko, A.E., Guenzl, P.M., Barlow, D.P., Pauler, F.M., 2013. Gene regulation by the act of long non-coding RNA transcription. BMC Biol. 11, 59.

Lee, D., Yu, M., Lee, E., et al., 2009. Tumor-specific apoptosis caused by deletion of the ERBB3 pseudo-kinase in mouse intestinal epithelium. J. Clin. Invest. 119, $2702-2713$.

Lee, H.S., Cho, H.J., Kwon, G.T., Park, J.H.Y., 2014. Kaempferol downregulates insulinlike growth factor-i receptor and ErbB3 signaling in HT-29 human colon cancer cells. J. Cancer Prev. 19, 161-169.

Lee, H., Lee, H., Chin, H., et al., 2014. ERBB3 knockdown induces cell cycle arrest and activation of Bak and Bax-dependent apoptosis in colon cancer cells. Oncotarget 5, 5138-5152.

Li, J.-H., Liu, S., Zhou, H., et al., 2014. starBase v2.0: decoding miRNA-ceRNA, miRNAncRNA and protein-RNA interaction networks from large-scale CLIP-Seq data. Nucleic Acids Res. 42, D92-D97.

Max Andersen, M.L.C., Hougaard, P., Pörksen, S., et al., 2014. Partial remission definition: validation based on the insulin dose-adjusted HbA1c (IDAA1C) in 129 Danish children with new-onset type 1 diabetes. Pediatr. Diabetes 15, 469-476.

Mirza, A.H., Kaur, S., Brorsson, C.A., Pociot, F., 2014. Effects of GWAS-associated genetic variants on IncRNAs within IBD and T1D candidate loci. PLoS One 9 e105723.

Olayioye, M.A., Neve, R.M., Lane, H.A., Hynes, N.E., 2000. The ErbB signaling network: receptor heterodimerization in development and cancer. EMBO J. 19, 3159-3167.

Onengut-Gumuscu, S., Chen, W.-M., Burren, O., et al., 2015. Fine mapping of type 1 diabetes susceptibility loci and evidence for colocalization of causal variants with lymphoid gene enhancers. Nat. Genet. 47, 381-386. http://dx.doi.org/ 10.1038/ng.3245.

Perocchi, F., Xu, Z., Clauder-Münster, S., Steinmetz, L.M., 2007. Antisense artifacts in transcriptome microarray experiments are resolved by actinomycin D. Nucleic Acids Res. 35, e128.

Pullen, T., Rutter, G., 2012. An islet-specific long non-coding RNA (lncRNA) expressed from the Pdx1 locus regulates Pdx1 activity. Diabetologia 55, 212.

Romanos, J., Wijmenga, C., 2009. Comment on: Barker et al. (2008) Two single nucleotide polymorphisms identify the highest-risk diabetes HLA genotype. Diabetes 57, 3152-3155, 2008. Diabetes 58:e1; author reply e2.

Rosenbloom, K.R., Sloan, C.A., Malladi, V.S., et al., 2013. ENCODE data in the UCSC Genome Browser: year 5 update. Nucleic Acids Res. 41, D56-D63.

Schaub, M.A., Boyle, A.P., Kundaje, A., et al., 2012. Linking disease associations with 
regulatory information in the human genome. Genome Res. 22, 1748-1759. Schulthess, F.T., Paroni, F., Sauter, N.S., et al., 2009. CXCL10 impairs beta cell function and viability in diabetes through TLR4 signaling. Cell Metab. 9, 125-139.

Stark, C., Breitkreutz, B.-J., Reguly, T., et al., 2006. BioGRID: a general repository for interaction datasets. Nucleic Acids Res. 34, D535-D539.

Stranger, B.E., Montgomery, S.B., Dimas, A.S., et al., 2012. Patterns of cis regulatory variation in diverse human populations. PLoS Genet. 8, e1002639.

Todd, J.A., Walker, N.M., Cooper, J.D., et al., 2007. Robust associations of four new chromosome regions from genome-wide analyses of type 1 diabetes. Nat. Genet. 39, 857-864.

Törn, C., Hadley, D., Lee, H.-S., et al., 2015. Role of type 1 diabetes-associated SNPs on risk of autoantibody positivity in the TEDDY study. Diabetes 64, 1818-1829.

Tsui, S., Dai, W., Lu, L., 2014. CCCTC-binding factor mediates effects of glucose on beta cell survival. Cell Prolif. 47, 28-37.

Wang, H., Jin, Y., Reddy, M.V.P.L., et al., 2010. Genetically dependent ERBB3 expression modulates antigen presenting cell function and type 1 diabetes risk. PLoS One 5, e11789.

Westra, H.-J., Peters, M.J., Esko, T., et al., 2013. Systematic identification of trans eQTLs as putative drivers of known disease associations. Nat. Genet. 45 $1238-1243$.

Wilusz, J.E., Sunwoo, H., Spector, D.L., 2009. Long noncoding RNAs: functional surprises from the RNA world. Genes Dev. 23, 1494-1504.

Winkler, C., Krumsiek, J., Lempainen, J., et al., 2012. A strategy for combining minor genetic susceptibility genes to improve prediction of disease in type 1 diabetes. Genes Immun. 13, 549-555.

Xie, C., Yuan, J., Li, H., et al., 2014. NONCODEv4: exploring the world of long noncoding RNA genes. Nucleic Acids Res. 42, D98-D103.

Xu, Z., Wei, W., Gagneur, J., et al., 2011. Antisense expression increases gene expression variability and locus interdependency. Mol. Syst. Biol. 7, 468. 